\title{
Análisis comparativo de las características fisicoquímicas del agua en la zona litoral, limnética y el caño de la ciénaga La Honda, Tanguí, Medio Atrato, Chocó, Colombia
}

\author{
Comparative analysis of water physicochemical features in the coastal \\ zone, limnetica and sewer of La Honda wetland, Tanguí, Medio Atrato, \\ Chocó, Colombia
}

\author{
Yirlesa Murillo Hinestroza', Lady Vargas Porras²
}

\section{Resumen}

La Honda, es una ciénaga principal tipo 1, conectada directamente con el rio Atrato a través de un caño. Se realizaron mediciones in situ de variables fisicoquímicas en la zona litoral, limnética y el caño, buscando determinar la calidad del recurso hídrico y el de conservación del ecosistema. Para esto, se seleccionaron 8 puntos de muestreo distribuidos en las tres zonas y se hicieron 4 monitoreos de variables como sólidos, turbiedad, nutrientes y temperatura con equipos multiparamétricos. Se encontró una temperatura promedio de $28^{\circ} \mathrm{C}$ y concentraciones de oxígeno disuelto en la zona litoral y limnética por encima de $4.4 \mathrm{mg} / \mathrm{l}$, la conductividad presentó valores inferiores a $12 \mu \mathrm{s} / \mathrm{cm}$, así como bajos niveles de sólidos disueltos que oscilaron entre 5.4 y $7 \mathrm{mg} / \mathrm{l}$, lo que se relacionó con la poca turbiedad en el agua (9.9 y 13.5 FAU). A partir de los datos arrojados y el análisis realizado en las zonas, se logró establecer el buen estado de conservación de la ciénaga y sus condiciones aptas para la preservación de la vida acuática, lo que convierte a este ecosistema en un objeto de preservación dada la importancia ecológica y ambiental de estos ambientes, tanto para la conservación de la biodiversidad como para la susbsistencia de las comunidades localizadas en su área de influencia y que hacen uso de los recursos naturales que alberga.

Palabras clave: Calidad del agua, Ciénaga la Honda, Ecosistemas, Medio Atrato, Variables fisicoquímicas

\begin{abstract}
The Honda is a major morass type 1, connected directly to the Atrato River through a pipe in it in situ physicochemical variables measurements were performed in the coastal zone, limnetic and spout, seeking to determine the quality of water resources and ecosystem conservation. To this, 8 sampling points distributed in three zones and four monitoring variables as solids, turbidity, temperature and nutrient multiparametric equipment were selected. An average temperature of $28^{\circ} \mathrm{C}$ and dissolved oxygen concentrations in the littoral and limnetic zone are above $4.4 \mathrm{mg} / \mathrm{l}$ was found, conductivity presented below $12 \mu \mathrm{s} / \mathrm{cm}$ values and low levels of dissolved solids ranging from 5.4 and $7 \mathrm{mg} / \mathrm{l}$, which was associated with low turbidity in water (9.9 and $13.5 \mathrm{FAU}$ ). From the data obtained and the analysis in the areas, it was established the good condition of the swamp and suitable for the preservation of aquatic life conditions, making this ecosystem an object of preservation
\end{abstract}

1 Ingeniera Ambiental, Instituto de Investigaciones Ambientales del Pacífico (IIAP), Quibdó, Chocó, Colombia.

e-mail: yirdavid@hotmail.com

2 Ingeniera Ambiental y Sanitaria, MSc Ciencias Ambientales, Investigadora Principal Componente Ambiental, Instituto de Investigaciones Ambientales del Pacífico (IIAP), Quibdó, Chocó, Colombia. e-mail: lvargas@iiap.org.co

Recibido: 13 de octubre de 2013

Aceptado: 4 de marzo de 2014 


\section{Bioetnia Volumen 11, 2014}

given the ecological and environmental importance of these environments, both for biodiversity conservation and for the subsistence of the communities located in its area of influence and make use of natural resources it contains.

Keywords: Ecosystems, Honda Wetlands, Medio Atrato, Physico-chemical variables, Water quality.

\section{Introducción}

Las ciénagas son ecosistemas altamente dinámicos, sujetos a una amplia gama de sistemas naturales que determinan su modificación en el tiempo aun en ausencia de factores de perturbación (Junk 1980). Debido a esto, todas y en especial las del Medio Atrato son benéficas no solo desde el punto de vista ecológico sino también a nivel socioeconómico, por sus múltiples funciones, valores y atributos, los cuales son esenciales tanto para los organismos presentes en ellas, como para las comunidades aledañas a la misma, porque se convierten no solo en un medio de subsistencia para estos, sino también en un ecosistema con buena conservación donde las comunidades, pescan a gran escala, la utilizan para la recreación pasiva y son importantes para la educación e investigación científica.

Al respecto, el Ministerio de Ambiente, Vivienda y Desarrollo Territorial (2007), señala que humedales como las ciénagas, representan numerosos beneficios relacionados con sus funciones ecológicas y ambientales, debido a que sirven de soporte de actividades productivas y socio-culturales y mantienen la biodiversidad.

Según Madrid (2007), estas son áreas estratégicas y de suma importancia por cumplir tres funciones básicas: 1) las físicas, como regulación del ciclo hídrico superficial y de los acuíferos, la retención de sedimentos, el control de erosión y la estabilización microclimática; 2) las químicas como la regulación de los ciclos de nutrientes (retención, filtración y liberación) y la descomposición de biomasa terrestre como base de la productividad de los sistemas acuáticos; 3) las bio-ecológicas como la productividad biológica, la estabilidad e integridad de los ecosistemas asociados y la retención de dióxido de carbón.

De igual forma las ciénagas disminuyen o mitigan los efectos producidos por el calentamiento global, porque estos ambientes acuáticos sirven de sumidero al 40\% del carbono que se genera en el planeta (Ariza et al. 2006), es decir, eliminan importantes cantidades de gases de efecto invernadero, además de que transfieren nutrientes al suelo con minerales provenientes del drenaje de la cuenca e igualmente equilibran el pH y con ello la apropiada relación entre acidez y alcalinidad, lo cual es fundamental para la vitalidad de los bioecosistemas correspondientes y adicionalmente mantienen múltiples formas de vida silvestre.

Se evaluó la calidad del agua del ecosistema mediante la medición y análisis de variables fisicoquímicas que dejaron al descubierto, el estado de conservación del recurso como hábitat para la biota acuática y como fuente de servicios paras las comunidades asentadas en su área de influencia.

\section{Materiales y métodos}

Área de estudio. En el corregimiento de Tanguí, que hace parte del Medio Atrato ubicado por vía fluvial a 30 minutos de Quibdó, capital del departamento del Chocó, se encuentra la ciénaga La Honda a $5^{\circ} 52^{\prime} 27.3^{\prime \prime} \mathrm{N}$ y $76^{\circ}$ $44^{\prime} 48.9^{\prime \prime} \mathrm{W}$ (Figura 1), que se encuentra constituida por un espejo de agua dulce de aproximadamente 4 metros de profundidad en su zona central y se caracteriza por presentar aguas con poca turbiedad, presencia de vegetación sumergida, flotante y sobre la ribera de la misma, además de un lecho de sedimento fangoso.

Se seleccionaron tres puntos de muestreo en zona litoral, dos en la zona limnética y tres en el caño, para un total de ocho puntos de muestreo previo a un recorrido por la Ciénaga (Tabla 1). En cada punto se georeferenció y se empleó una

Tabla 1. Distribución y localización de los puntos de muestreo

\begin{tabular}{|c|c|c|}
\hline Punto & Descripción & Coordenadas \\
\hline 1 & Unión entre el caño y el río Atrato & 5053'39.6"'- 76043'47.4" \\
\hline 2 & Zona media del caño & 5052'35.3"'- 76044'29.4" \\
\hline 3 & Unión entre el caño y la ciénaga & 5052'27.3"'- 76044'48.9"' \\
\hline 4 & Zona de la ribera (litoral) de la ciénaga & 5052'34.1"'- 76044'53.7"' \\
\hline 5 & Zona de la rivera (litoral) de la ciénaga & 5052 '43.9'"- 76045'43.9" \\
\hline 6 & Zona de la rivera (litoral) de la ciénaga & 5052'29.3"'- 76044'54.9"' \\
\hline 7 & Zona central (limnética) de la ciénaga & 505'31.8”- 76044'55.1"' \\
\hline $7 \mathrm{~A}$ & Zona central (limnética) de la ciénaga a $2 \mathrm{~m}$ de profundidad & 505'31.8'"- 76044'55.1" \\
\hline
\end{tabular}




\section{Características fisicoquímicas del agua. Y Murillo, L Vargas}

botella muestradora tipo Van Dorn para la recolección de muestras a diferentes profundidades. Se hicieron mediciones in situ de variables fisicoquímicas como temperatura, conductividad, sólidos disueltos, sólidos suspendidos, turbiedad, oxígeno disuelto, $\mathrm{pH}$, nitrato, nitrito, sulfato y fosfato, utilizando un colorímetro portátil HACH 850 y un multiparámetro YSI PROFESIONAL PLUS QUICK 1700/1725. Los resultados obtenidos fueron comparados con los estándares de calidad para la preservación de la fauna y la flora en diferentes países y con los resportes arrojados por otras investigaciones de calidad de agua en ecosistemas cenagosos.

\section{Resultados y discusión}

Análisis de variables fisicoquímicas en el caño, la zona litoral y limnética de la ciénaga. Los resultados del análisis fisicoquímico realizado in situ en la zona litoral, limnética y el caño de la ciénaga La Honda, aparecen en la Tabla 2. Se encontró que en las tres zonas muestreadas, el pH y la temperatura tuvieron variaciones casi nulas, mostrando, valores promedios de $5.9,5.5,5.6$ y $27.1,28.1$ y $26.9^{\circ} \mathrm{C}$ respectivamente, panorama que indica aguas moderadamente ácidas y que no afectan el desarrollo de la biota acuática, lo que concuerda con López (1991), quien argumenta que valores de $\mathrm{pH}$ en estos rangos favorecen la vida acuática y por lo tanto suponen una fuente hídrica en buen estado.

Las variaciones de temperatura encontradas en las zonas monitoreadas, indican aguas cálidas con una permanencia que favorece significativamente el desarrollo de la fauna y flora, teniendo en cuenta que la temperatura es un factor muy condicionante de la vida acuática, porque su variación no solo afecta la solubilidad de gases como el oxígeno disuelto, quien por su parte es una variable determinante en la presencia o ausencia de grupos biológicos acuáticos en un ecosistema, sino también de variables como el pH y la conductividad entre otras, lo que concuerda con lo afirmado por Mitchell et al. (1991), quienes afirman que la temperatura influye en la solubilidad de las sales y los gases, al igual que en la disociación de las sales disueltas y por lo tanto en el pH y la conductividad eléctrica de agua.

La conductividad osciló entre 8.4 y $20 \mu \mathrm{s} / \mathrm{cm}$ con un valor promedio de $13.6 \mu \mathrm{s} / \mathrm{cm}$ y los sólidos disueltos oscilaron y 4.9 y $12.5 \mathrm{mg} / \mathrm{l}$ con un valor promedio de $8.4 \mathrm{mg} / 1$, presentando los valores más altos en la zona correspondiente al caño, lo que pudo estar relacionado con una elevada cantidad de sustancias disueltas y material particulado en proceso de descomposición, teniendo que esta zona esta más cerca al río Atrato, lugar donde hay presencia de asentamientos humanos y por lo tanto el desarrollo de diferentes actividades que dan lugar al aporte de contaminantes, que junto con los iones disueltos en el agua generan un incremento

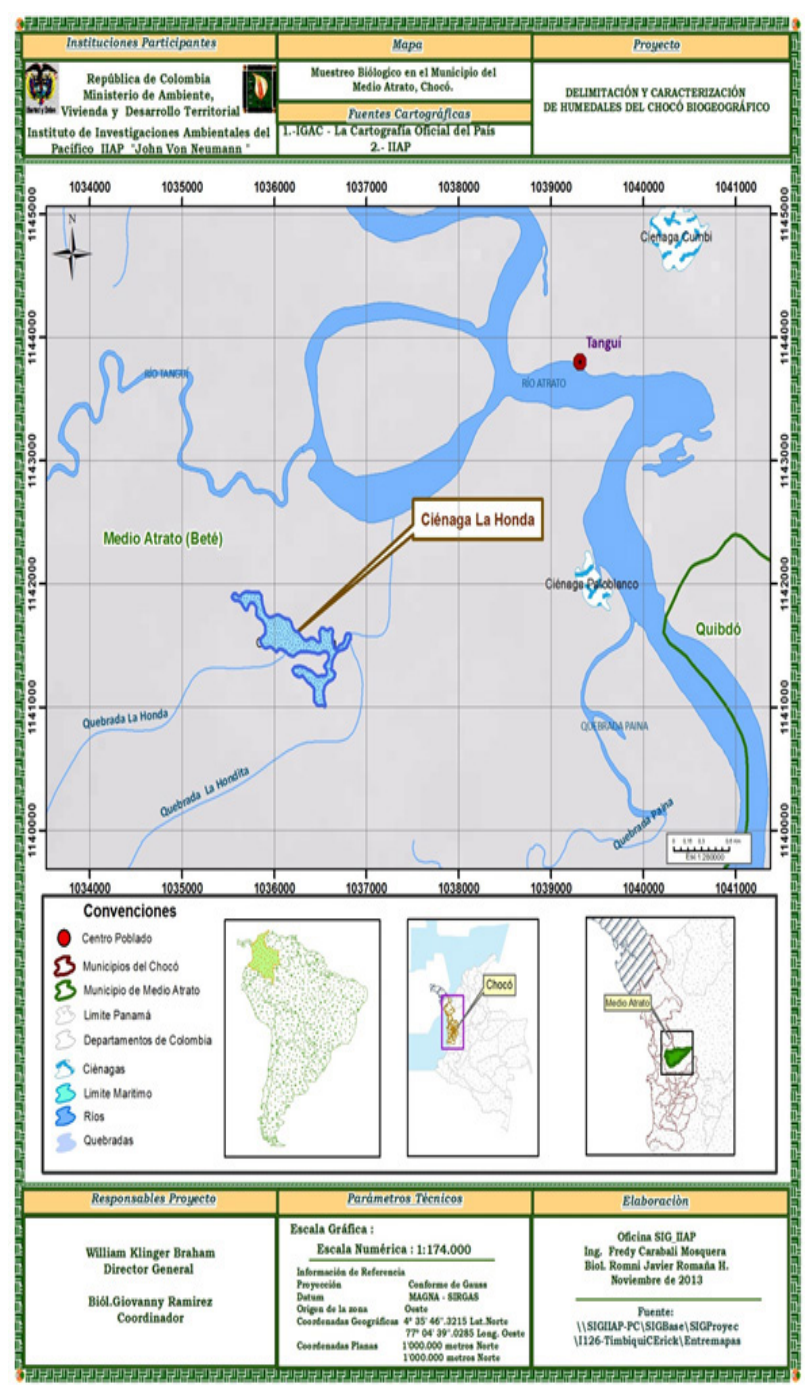

Figura 1. Ubicación de la ciénaga La Honda.

de los valores de conductividad encontrados, evidenciando que esta zona de intercambio constituye un punto crítico en la calidad del agua de todo el sistema. Al respecto, Crites y Tchobanoglous (2000), afirman que un aumento en la concentración de iones disueltos en el agua, provoca un aumento en la conductividad de la misma, lo que puede afectar la biota acuática en concentraciones elevadas.

Contrario a esto, la zona litoral y limnética de la ciénaga, presentaron una reducción en la conductividad y los sólidos disueltos, mostrando un mejor estado de calidad del agua sobre todo en su zona limnética como consecuencia de la poca presencia de contaminantes que puedan favorecer el incremento de estas variables en el agua, lo que resulta benéfico para el desarrollo de la fauna y flora al interior de la misma.

Por otro lado, las variables relacionadas con la presencia de material suspendido en el agua presentaron un comportamiento similar en las zonas muestreadas, porque 
Bioetnia Volumen 11, 2014

Tabla 2. Valores obtenidos in situ de variables fisicoquímicas en diferentes zonas de la ciénaga La Honda

\begin{tabular}{|c|c|c|c|c|c|c|c|c|c|c|c|}
\hline \multirow{3}{*}{ Parámetros } & \multicolumn{4}{|c|}{ Caño } & \multicolumn{4}{|c|}{ Zona Litoral } & \multicolumn{3}{|c|}{ Zona Limnética } \\
\hline & & & & & & unto & & & & & \\
\hline & 1 & 2 & 3 & * & 4 & 5 & 6 & * & 7 & $7 a$ & * \\
\hline \multicolumn{12}{|l|}{ Conductividad } \\
\hline$(\mu \mathrm{s} / \mathrm{cm})$ & 20.03 & 12.6 & 8.4 & 13.6 & 9.0 & 9.5 & 7.9 & 8.8 & 9.1 & 15.0 & 12.0 \\
\hline Temperatura (0C) & 26.8 & 26.9 & 27.8 & 27.1 & 28.0 & 28.3 & 28.2 & 28.1 & 28.3 & 25.6 & 26.9 \\
\hline \multicolumn{12}{|l|}{ Oxígeno disuelto } \\
\hline$(\mathrm{mg} / \mathrm{l})$ & 5.2 & 2.9 & 4.0 & 4.0 & 4.5 & 4.7 & 4.5 & 4.5 & 4.2 & 4.7 & 4.4 \\
\hline \multicolumn{12}{|l|}{ Sólidos disueltos } \\
\hline$(\mathrm{mg} / \mathrm{l})$ & 12.5 & 8.0 & 4.9 & 8.4 & 5.6 & 6.0 & 4.7 & 5.4 & 5.63 & 8.45 & 7.04 \\
\hline \multicolumn{12}{|l|}{ Sólidos suspendidos } \\
\hline$(\mathrm{mg} / \mathrm{l})$ & 135.0 & 15.0 & 16.6 & 55.5 & 10.6 & 11.0 & 6.6 & 9.4 & 7.66 & 3.5 & 5.5 \\
\hline $\mathrm{pH}$ & 6.5 & 5.7 & 5.5 & 5.9 & 5.6 & 5.6 & 5.4 & 5.5 & 5.5 & 5.7 & 5.6 \\
\hline Turbiedad (FAU) & 109 & 18.3 & 11 & 46.1 & 12.3 & 7.3 & 10.3 & 9.9 & 13 & 14 & 13.5 \\
\hline Sulfatos (mg/l) & 22.0 & 2.0 & 1.0 & 8.3 & 4.6 & 8.6 & 2.66 & 5.2 & 1.0 & 13.0 & 7.0 \\
\hline Fosfatos (mg/l) & 1.31 & 0.24 & 0.86 & 0.80 & 0.25 & 1.14 & 0.25 & 0.54 & 1.66 & 0.49 & 1.07 \\
\hline Nitratos (mg/l) & 0.66 & 0.50 & 0.57 & 0.57 & 0.51 & 0.46 & 0.41 & 0.46 & 0.45 & 0.58 & 0.51 \\
\hline Nitritos (mg/l) & 0.42 & 0.031 & 0.025 & 0.15 & 0.027 & 0.033 & 0.039 & 0.033 & 0.016 & 0.086 & 0.051 \\
\hline
\end{tabular}

* Promedio 1. Unión caño y río Atrato 8:50 am 2. Zona media del caño 9:30 am 3 . Unión entre el caño y la ciénaga 10:30 am 4. Zona de la ribera de la ciénaga 11:05 am 5 . Zona de la ribera de la ciénaga 11:45 am $\quad 6$. Zona de la ribera de la cienaga 12:17 am 7. Zona central de la ciénaga 12:45 am 7a. Zona central de la ciénaga, $2 \mathrm{~m}$ de profundidad, 1:30 pm

en el caño, la turbiedad varió entre 11 y 109 FAU y los sólidos suspendidos oscilaron entre 16.6 y $135 \mathrm{mg} / \mathrm{l}$ respectivamente, mientras que en las zonas litoral y limnética estas variables alcanzaron promedios entre 5.5 y $5.6 \mathrm{FAU}$ y 9.4 y $5.5 \mathrm{mg} / \mathrm{l}$ respectivamente. Esta situación puede estar relacionada también con la conexión entre el caño y el río Atrato, que recibe numerosos aportes de vertimientos sólidos y líquidos tanto de origen natural como antrópico, por la gran cantidad de asentamientos humanos y actividades productivas agrícolas, mineras y de transporte localizadas sobre su cauce y márgenes, incrementando los valores de turbiedad, el nivel de sólidos suspendidos en el agua, los cuales por las dinámicas de flujo son transportados a través del río hasta al caño, quien hace las veces de filtro natural del agua, permitiendo que al interior de la ciénaga el material suspendido llegue en menor proporción, lo que puede explicar que en la zona de intercepción río-caño estas variables presenten su máxima concentración y hacia el interior de la ciénaga (litoral y limnética) los valores se reduzcan considerablemente. Una situación similar reportó Betancourt y Ramírez (2005) para la ciénaga Grande de Santa Marta donde encontraron que las aguas que ingresan a ella por la boca del caño Aguas Negras presenta niveles superiores de contaminantes que al momento de salir a través de la boca del Caño Grande, obteniendo diferencias cercanas a $50 \%$ de remoción, lo que atribuyeron a que a través de los caños alimentadores se observan altas tasas de sedimentación, constituyendo la principal forma de remoción de partículas en la columna de agua. En relación con esto Barbanti (1992) ha indicado que los flujos lentos por los caños hacen que los equilibrios iónicos cambien, facilitando la sedimentación y por ende la disminución de los compuestos asociados con las partículas suspendidas.

Las concentraciones de oxígeno disuelto arrojaron valores promedios de $4 \mathrm{mg} / \mathrm{l}$ para el caño, $4.5 \mathrm{mg} / \mathrm{l}$ para la zona litoral y $4.4 \mathrm{mg} / \mathrm{l}$ en el área limnética, lo que muestra valores que si bien no son elevados, indican aguas oxigenadas que favorecen el desarrollo de la vida acuática, evidenciando que la ciénaga presenta condiciones estables para el mantenimiento de las comunidades biológicas que habitan en ella. Es de anotar que este decrecimiento en la concentración del oxígeno desde el interior del cuerpo de agua hacia el caño, corrobora los resultados obtenidos para variables anteriores como sólidos, conductividad y turbiedad que presentaron un comportamiento inverso, debido a que las sustancias disueltas en el agua consumen y reducen la 


\section{Características fisicoquímicas del agua. Y Murillo, L Vargas}

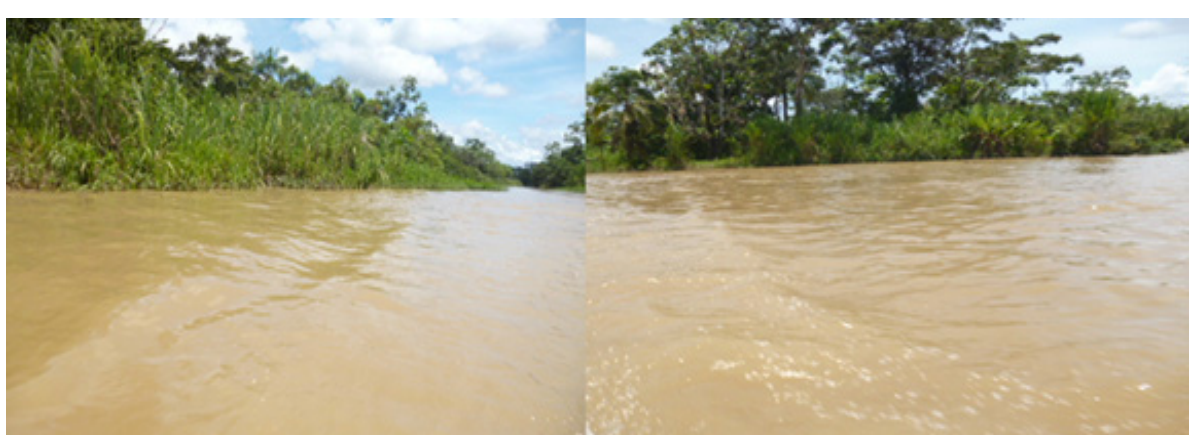

Figura 2. Turbiedad y turbulencia en el punto 1 unión entre el caño y el río Atrato

disponibilidad de oxígeno, haciendo que en el caño sean menor, teniendo en cuenta que por su conexión directa con el río Atrato, se ve influido por la gran cantidad de material disuelto y la alta turbiedad localizada en el mismo (Figura2).

No obstante, todos los valores de oxígeno disuelto encontrados, favorecen el desarrollo óptimo de fauna y flora al interior del agua, porque autores como el Ministerio de Agricultura (1984), Lenntech (2007) y Stevens Institute of Technology (2006), expresan que aguas con concentraciones $>4 \mathrm{mg} / \mathrm{l}$ son benéficas para el desarrollo de las comunidades biológicas, de ahí que en términos generales, La Honda constituya un ecosistema con características fisicoquímicas aptas para una buena actividad biológica, debido a la disponibilidad de oxígeno disuelto encontrada en casi todos los puntos de muestreo. A este respecto Malina (1996) ha indicado que disminuciones repentinas o graduales en el oxígeno disuelto pueden ocasionar cambios bruscos en el tipo de organismos acuáticos, por ejemplo insectos acuáticos sensibles a un nivel bajo de oxígeno disuelto, pueden ser reducidas sus poblaciones.

En cuanto a los nutrientes (sulfatos, fosfatos, nitratos y nitritos), se encontraron valores promedios de 8.3, $0.80,0.57$ y $0.15 \mathrm{mg} / 1$ respectivamente, para la zona del caño, lo que demuestra aguas con cierto grado de oxigenación, no muy contaminadas y por lo tanto aptas para el desarrollo de la biota acuática. Comportamiento similar ocurrió en la zona litoral y limnética de la ciénaga, en la cual dichos nutrientes arrojaron concentraciones promedio más bajas de $5.2,7 \mathrm{mg} / 1$ para los sulfato, $0.54,1.07 \mathrm{mg} / \mathrm{l}$ de fosfatos, $0.46,0.51 \mathrm{mg} / \mathrm{l}$ de nitratos y $0.03,0.05 \mathrm{mg} / \mathrm{l}$ de nitritos, presentando los valores más bajos en la zona limnética, esto posiblemente por la poca presencia de aportes de materia orgánica provenientes de la vegetación que puedan causar un incremento de estas variables especialmente en los nitratos y nitritos. Al respecto autores como Kadlec y Knight (1996), Stumm y Morgan, (1981), indican que estos elementos generalmente son iones que existen de manera natural, en bajas concentraciones y no superan los $0.1 \mathrm{mg} / 1$, lo que evidencia el bajo nivel de intervención de este ecosistema.

Sumado a esto, los valores de sulfato encontrados en las zonas evaluadas, no representan una afectación para el desarrollo de la vida acuática, lo que coincide con lo expresado Moreno (2013), quien manifiesta que las aguas dulces contienen entre 2 y $150 \mathrm{ppm}$ y se saturan a unos $1.500 \mathrm{ppm}$, de ahí que no exista un límite o rango de concentración óptimo de sulfatos para la fauna acuática. Asimismo, las concentraciones de fosfatos reportados, resultan normales en este tipo de ecosistemas, por lo que no interfieren en la actividad productiva de la fuente hídrica, teniendo en cuenta que estos existen naturalmente en forma disuelta y son eliminados del suelo por efecto del agua o el viento; permite inferir que las concentraciones encontradas corresponden a aportes de origen natural por el intercambio entre los sustratos y el recurso hídrico.

Estado del agua como hábitat para el establecimiento de organismos. Un análisis del estado del agua como hábitat para la biota acuática en la ciénaga La Honda, permite expresar que tanto la zona del caño, como la litoral y limnética, presentan condiciones fisicoquímicas que favorecen el desarrollo de las especies en el ecosistema (Tabla 3), lo que está relacionado con la poca intervención antrópica del cuerpo de agua, porque su uso está orientado a la pesca y al transporte de los recursos extraídos de los bosques del área de influencia. Sin embargo, cabe mencionar que la zona del caño presenta cierto nivel de afectación como resultado de su interconexión e intercambio hídrico con el río Atrato que recibe mayores aportes de origen antrópico y que sugieren el incremento de material suspendido y disuelto en el agua.

En este sentido, la Tabla 3 evidencia que todos los parámetros analizados se encuentran dentro de los límites establecidos por diferenres estándares de calidad de agua para conservación de la vida acuática. Aunque el pH se encontró ligeramente ácido en las 3 zonas, los valores obtenidos se encuentran muy cerca de los limítes permisibles, lo que podría ser indicativo de la presencia de ciertos niveles de materia orgánica o algunos iones durante el muestreo que favorecieron este resultado. Sin embargo, el conjunto de variables analizadas demuestra el buen estado de salud del ecosistema, lo que denota además, la importancia de conservación del mismo, porque se encuentra inmerso en todo un 
Bioetnia Volumen 11, 2014

Tabla 3. Comparación de las zonas muestreadas en la ciénaga La Honda con estándares de calidad de agua para la preservación de la biota acuática

\begin{tabular}{|c|c|c|c|c|c|c|c|c|}
\hline \multirow[t]{3}{*}{ Parámetro } & \multicolumn{6}{|c|}{ Criterios de calidad de agua para conservación de biota acuática por país } & \multirow{2}{*}{\multicolumn{2}{|c|}{$\begin{array}{c}\text { Datos de muestreo } \\
\text { Zona }\end{array}$}} \\
\hline & \multirow[t]{2}{*}{ Panamá } & \multirow[t]{2}{*}{ Argentina } & \multirow[t]{2}{*}{ Uruguay } & \multirow[t]{2}{*}{ Colombia } & \multirow[t]{2}{*}{ México } & \multirow[t]{2}{*}{ Caño } & & \\
\hline & & & & & & & litoral & limnérica \\
\hline $\mathrm{pH}$ & $5.0-9.0$ & $6.5-8.5$ & $6.0-9.0$ & $4.5-9.0$ & $6.0-8.0$ & 5.9 & 5.5 & 5.6 \\
\hline Oxígeno disuelto & & & & $4.0 \mathrm{mg} / \mathrm{l}$ & $5.0 \mathrm{mg} / \mathrm{l}$ & $4.0 \mathrm{mg} / \mathrm{l}$ & $4.5 \mathrm{mg} / \mathrm{l}$ & $4.4 \mathrm{mg} / \mathrm{l}$ \\
\hline Nitratos & $<200 \mathrm{mg} / \mathrm{l}$ & $<30 \mathrm{mg} / \mathrm{l}$ & & & & $0.57 \mathrm{mg} / \mathrm{l}$ & $0.46 \mathrm{mg} / \mathrm{l}$ & $0.51 \mathrm{mg} / \mathrm{l}$ \\
\hline Nitritos & $<0.6 \mathrm{mg} / \mathrm{l}$ & & & & & $0.15 \mathrm{mg} / \mathrm{l}$ & $0.03 \mathrm{mg} / \mathrm{l}$ & $0.051 \mathrm{mg} / \mathrm{l}$ \\
\hline Sulfatos & & $<500 \mathrm{mg} / \mathrm{l}$ & & & $0.005 \mathrm{mg} / \mathrm{l}$ & $8.3 \mathrm{mg} / \mathrm{l}$ & $5.2 \mathrm{mg} / \mathrm{l}$ & $13.5 \mathrm{mg} / \mathrm{l}$ \\
\hline Fosfatos & & & $0.025 \mathrm{mg} / \mathrm{l}$ & & $0.025 \mathrm{mg} / \mathrm{l}$ & $0.80 \mathrm{mg} / \mathrm{l}$ & $0.54 \mathrm{mg} / \mathrm{l}$ & $7.0 \mathrm{mg} / \mathrm{l}$ \\
\hline
\end{tabular}

sistema hídrico conformado por el río Atrato, las ciénagas de su zona inundabley los ambientes boscosos interconectados, que entre si soportan una gran biodiversidad que depende del mantenimiento de los flujos de agua y nutrientes por todos estos elementos y que constituye el principal medio de obtención de recursos económicos y alimenticios para las comunidades localizadas en su área de influencia.

\section{Conclusiones}

El análisis de variables fisicoquímicas en las zonas monitoreadas, permite concluir que todas y sobre todo la zona litoral y limnética de la ciénaga, presenta un recurso hídrico con condiciones favorables tanto para la biota acuática como para la oferta de bienes y servicios a las comunidades aledañas a esta. Estas características la convierten en uno de los principales medios de sustento para una población que no solo ve en ella un ecosistema apto para el desarrollo de las comunidades biológicas en su interior y en ecosistemas conexos, sino que también la utilizan como fuente de abastecimiento de agua como se evidenció en campo. Los resultados obtenidos permiten considerar la zona litoral y limnética de La Honda, como un ecosistema no solo de gran importancia ecosistémica por su ubicación estratégica dentro del valle inundable del río Atrato, sino de gran interés socioeconómico, porque soporta la pesca como principal actividad de sostenimiento de las comunidades aledañas.

Conforme a ello, es importante que se continúen realizando estudios encaminados con la implementación de estrategias que permitan la conservación de la misma. Se recomienda realizar monitoreo de calidad de agua, no solo a la ciénaga de estudio sino también a zonas que directa $\mathrm{o}$ indirectamente intervienen en la productividad de esta, como el caño y su conexión con el río Atrato, en aras de identificar posibles aspectos que atenten contra la integridad, el bienestar de las comunidades biológicas al interior de la misma y en general con la productividad del ecosistema.

\section{Literatura citada}

Ariza AI, Ramírez AI, Moncaleano AM, Córdoba PM. 2006. Los humedales como herramienta para el alivio de la pobreza. Tercera Jornada Académica. [consulta 3 de octubre de 2013]. Disponible en: http: ://www. ramsar.org/pdf/wwd/6/wwd2006_rpts_colombia01.pdf

Barbanti A, Frascari F, Paltrinieri D, Rosso G. 1992. Transport of nutrients in rivers: investigations on the river Po (Italy). Sci Total Envir. 92 (suppl): $337-44$.

Betancourt J, Ramírez G. 2005. Estudio de los procesos relacionados con la presencia de plaguicidas organoclorados en la ciénaga Grande de Santa Marta. Bol INVEMAR. 34 (1): 121-39.

Crites R, Tchobanoglous G. 2000. Sistemas de manejo de aguas residuales para núcleos pequeños y descentralizados. Bogotá: McGrawHill; $701 \mathrm{pp}$.

Junk W. 1980. Areas inundaveis. Um desafio para a limnologia. Acta Amazonica. 10: 775-995.

Kadlec RH, Knight RL 1996. Treatment wetlands. En: Vymazal J (ed.). Transformactions of nutrients in natural and construted wetlands. Boca Raton: CRC Press/Lewis Publishers; 893 pp.

Lenntech. 2007. Agua residualy purificación del aire. [consulta 16 de septiembre de 2007]. Disponible en: http://www.lenntech.com/espanol

López P. 1991. Química sanitaria 1. Manual de laboratorio. Cali: Publicaciones Facultad Ingeniería, Universidad del Valle.

Malina JF. 1996. Water quality. In: Mays L (eds.). Water resources handbook. New York: McGraw-Hill. pp. 8.3-8.49.

Mitchell M, Stapp W, Bixby K. 1991. Manual de campo de Proyecto del Río: una guía para monitorear la calidad del agua en el río Bravo. $2^{\mathrm{a}}$ ed. New Mexico: Proyecto del Río; 200 pp.

Madrid CA. 2007. Conservación de la biodiversidad de importancia mundial a lo largo de la costa chilena. Limnología de la desembocadura del río Copiapó como parte del Proyecto GEF "Conservación de la biodiversidad de importancia mundial a lo largo de la costa chilena". [consulta: 3 de octubre de 2013]. Gobierno de Chile/Proyecto GEF-Marino/PNUD. Disponible en: http://www.fundacionbiomar.cl/wp-content/uploads/ Libro-areas-marinas-20-pagsOK.pdf

Ministerio de Agricultura. 1984. Decreto 1594 de 1984. Bogotá: MinAgricultura; $55 \mathrm{pp}$.

Ministerio de Ambiente, Vivienda y Desarrollo Territorial. 2007. Resolución $N^{\circ}$ 978. [consulta noviembre 10 de 2010]. Disponible en: http://www. minambiente.gov.co/documentos/res_0978_040607.pdf

Moreno A. 2013. El agua. Calidad y contaminación. Parámetros químicos de calidad de las aguas. Cloruros y sulfatos. [consulta: junio 13 de 2013]. Disponible en: http://www.emagister.com/curso-agua-calidad-contaminacion-1-2/parametros-quimicos-calidad-aguas-cloruros-sulfatos

Stumm WS, Morgan JJ. 1981. Aquatic chemistry. An introduction enphasizing chemical equilibrium in natural water. New York, London, Sydney, Toronto: Ed. Wiley-Interscience; pp. 583.

Stevens Institute of Technology (SIT). 2006. Demanda biológica de oxígeno. [consulta 18 de septiembre de 2007]. Disponible en: http://www. $\mathrm{k} 12$ science.org 